\title{
¿Confusión de géneros? El ejemplo de la recepción del "Señor de los anillos" niñas y niños ${ }^{7}$
}

\section{Gender confusion? The case of the reception of "Lord of the Rings" in girls and boys}

\section{Christine Détrez}

Ecóle Normale Supérieure-LSH, Lyon

christine.detrez@ens-Ish.fr

\section{Resumen}

El estudio aborda, desde una perspectiva sociológica, las condiciones de recepción del filme El señor de los Anillos en audiencias juveniles, explorando en particular las posibles articulaciones entre el género de las audiencias y el género (formato) del producto audiovisual. De esta manera, examina el modo como estos factores inciden en la generación de expectativas, la construcción de roles, la ritualización de las percepciones y la elaboración de estereotipos.

Palabras clave: Sociología de la recepción, audiencias juveniles, El señor de los Anillos.

\section{Abstract}

The study tackles, from a sociological perspective, the reception conditions of the film "The Lord of the Rings" in teen audiences, exploring the possible links between the gender of audiences and genre (format) of the audiovisual product. Thus, examinites the way these factors influence the generation of expectations, the construction of roles, the ritualization of perceptions and the development of stereotypes.

Keywords: Sociology of the reception, teen audiences, The Lord of the Rings.

\section{Introducción}

La sociología de la cultura parece estar, en Francia, en un cambio de dirección de su propia historia: después de años de investigaciones cuantitativas y medidas estadísticas de dichas

7. Traducido desde el francés por Rafael del Villar Muñoz. 
prácticas, las interrogaciones surgen, vinculadas seguramente con una cierta desilusión, e incluso un desaliento por sus resultados: las desigualdades en materias de prácticas culturales se mantienen, y la democratización cultural parece ser un milagro inaccesible.

¿Es decir, es necesario abandonar las encuestas estadísticas, y la puesta en perspectiva de los determinismos sociales que obstaculizan el acceso a la cultura? Por supuesto que no. Por siempre eterna y cansante que puede parecer esta comprobación, es esencial colocarla como fondo y decorado de cualquier otra perspectiva de investigación. Pero denunciar el peso de los datos estructurales no impide cambiar, al mismo tiempo, de foco, y de completar el análisis de las prácticas con aquellas de la recepción. Lejos de oponerse a los estudios cuantitativos de las prácticas, hasta se puede profitar de ellos ...

Así, el estudio de las adaptaciones mediáticas de obras literarias permite pedir prestadas varias vías analíticas. Verdaderos productos "híbridos", estas adaptaciones se sitúan, en efecto, en el cruce entre la literatura, el cine, la televisión e incluso de la música. Así "El Señor de los Anillos fue adaptada al cine. En la televisión ha tenido lugar la moda, después de los años olvidados del fin de la ORTF, donde se adaptaban clásicos como Zola, Balzac, Hugo o Dumas. Otra vuelta en su funcionamiento es que las comedias musicales han agotado su repertorio clásico, como Nuestra Dama de París, Romeo y Juliette, etc.

La adaptación mediática de un clásico literario permite interrogarse sobre las recepciones puestas en juego, y en el mismo movimiento, interrogarse sobre ciertas certezas de la sociología de la cultura: ¿la mediatización de una obra no viene a perturbar las recepciones habitualmente observadas, en términos de legitimidad y en términos de la recepción de géneros? Es este segundo punto, él que yo deseo desarrollar aquí, a partir del análisis de la recepción del filme "El Señor de los Anillos"

\section{Géneros y prácticas de lectura}

La correlación entre género (sexo) y género (preferencia de formatos) emerge de las investigaciones estadísticas francesas, y, en particular, de los trabajos realizados sobre las prácticas de lectura: si se hace referencia a las Prácticas culturales de los Franceses (Donnat, 1997) un $3 \%$ de los hombres declaran leer generalmente ${ }^{9}$ novelas sentimentales, contra un $23 \%$ de las mujeres ( $4 \%$ de los hombres y $16 \%$ de las mujeres biografías novelescas), pero un $28 \%$ de los hombres dicen leer generalmente novelas de ciencia ficción o fantástica, contra un 9\% de las mujeres.

En cuanto a las lecturas de las adolescentes, de la misma manera, ciertos géneros pueden ser codificados por su elección más bien masculinas o más bien femeninas: hacia el polo

8. La interrogación en términos de legitimidades culturales ha sido desarrollada a partir de comedias musicales y teleseries adaptadas de clásicos literarios: ver Détrez, 2003.

9. Se puede poner en interrogante la pertinencia de la pregunta « leer más a menudo ». 
femenino, las novelas clásicas ( $52 \%$ de las jóvenes y un 30\% de los jóvenes han citado al menos un título, los testimonios con un 30\% de las muchachas y un 3\% de los muchachos, las novelas psicológicas con un $28 \%$ de las muchachas y un $12 \%$ de los muchachos); los best-sellers con un $11 \%$ las jóvenes y a penas un $1 \%$ de los jóvenes ${ }^{10}$ ).

Ciertos títulos son plebiscitados por los muchachos e ignorados por las muchachas,e inversamente: en la encuesta longitudinal anteriormente citada, ningún muchacho declaró "Jamás sin mi hija. Sólo tres muchachas (sobre 4000 respuestas recogidas en 4 años...) citaron al Señor de los Anillos. Esta separación es corroborada por los datos de una encuesta pasada en el año 2002 ante 1000 adolescentes de Drôme-Ardèche: cuando se les pide el último libro leído, sólo las muchachas respondieron La Hierba azul (12 citas), El Diario de Bridget Jones (9), o Las mujeres en blanco (4). Únicamente los muchachos declararon a Gaston Lagaffe (12), Blueberry o Joe Bar Equipo (4) ${ }^{11}$. A la cuestión relativa al libro que "los habría marcado", si el acuerdo se hace sobre Harry Potter, algunas respuestas también son muy sexuadas:

\begin{tabular}{ccc}
\hline Título que lo ha marcado & Muchachos & Muchachas \\
\hline Harry Potter & 44 & 48 \\
Yo, Cristina F., 13 años, & 3 & $\mathbf{6 5}$ \\
drogada, prostituida. & & \\
El Señor de los Anillos & $\mathbf{5 5}$ & 13 \\
El Pequeño Príncipe & 18 & 20 \\
La hierba azul & 1 & $\mathbf{3 0}$ \\
El diario de Ana Frank & 2 & $\mathbf{2 4}$ \\
Titeuf & 16 & 7 \\
Carne de gallina & 16 & 5 \\
Colmillo Blanco & 11 & 3 \\
Un saco de bolas & 7 & 6 \\
Mi bello naranjo & 4 & 9 \\
Jamás sin mi hija & 1 & $\mathbf{1 2}$ \\
Los diez pequeños negros & 6 & 6 \\
\hline
\end{tabular}

Ciertos títulos son, incluso, exclusivamente citados por las muchachas (Martina: 9; Las desgracias de Sophie: 7; El precio de la libertad, Carrie: 6; Las cuatro hijas del doctor March, Yo tenía doce años: 5; Love story, La vida en retroceso, $Y$ si era verdadero: 4) o por muchachos (Las hormigas, Lucky Luke, Lanfeust: 6; Star Wars: 5; El libro de las estrellas, El globo de oro, Bilbo el Hobbit, Blueberry: 4).

Sin embargo, tales clasificaciones deben matizarse. Por una parte, se basan básicamente en un método de razonamiento mayoritario: ciertamente, los muchachos son bien menos numerosos que las muchachas en citar testimonios o novelas sentimentales.

Sin embargo, sin incluso evocar el sesgo clásico en sociología de la lectura que hace que no se cite más que lo que se considera digno de sí, y la reticencia masculina "a reconocer"

10. La problemática está abierta. Los títulos recogidos han sido codificados a posteriori (Baudelot et al., 1999).

11. Se juega sin duda aquí igualmente un efecto de legitimidad que hace que las jóvenes sean menos proclives a declarar un título de dibujo animado cuando se les pregunta una cuestión relativa al « libro ». 
lecturas rosas, puede ser interesante observar que hay, a pesar de todo, al interior de los cuadros estadísticos, muchachos que leen novelas sentimentales y muchachas seducidas por las novelas de aventura o de ciencia ficción. Además, es importante destacar que la diferencia inducida por el sexo en las elecciones de lectura se reducen a medida que aumenta la escolarización, y esto se ve notablemente en el paso al colegio secundario respecto a lo que era inducido en la situación escolar. De "dime lo que lees, y yo te diré sí eres niña o muchacho" se pasa a "yo te diré en qué clase tu estás y si tu estás bien en la escuela...” (Baudelot et al., ibid.)

Por otra parte, se podría temer que, encuesta por encuesta, no se sedimentan los resultados esperados y no se esencializa de alguna manera un supuesto gusto de género. Lo anterior es un ejemplo: entre remarcar un debilitamiento de los gustos vinculados al género en favor de los gustos vinculados a la posición escolar, y deducir de esto un efecto "perverso" de la escuela que vendría a "perturbar" los gustos anteriores, (z”naturales”?), el paso puede rápidamente cruzarse. Las nociones mismas de lectura "ordinaria" y de lectura "sabia", a menudo utilizados para diferenciar las maneras de leer, pueden por lo pronto prestarse a tales interpretaciones: la lectura "ordinaria", fundada sobre la emoción, la identificación, la experiencia ético practica, etc., correspondería en efecto a un estado primero del placer de leer, transformado - ipervertido? - por la institución escolar en la lectura sabia, en cuanto que ella está basada en el retroceso, la interpretación. Pero estos gustos "naturales" no son ellos mismos más que los efectos de una socialización anterior, más familiar que escolar.

Por último, es necesario igualmente tener en cuenta, en la formación de los gustos, el papel de la socialización de los amigos, y mediática. Así pues, la comparación de los datos recogidos en la investigación longitudinal (cuya recolección se había terminado en 1996) y la encuesta del 2002 en Drôme Ardèche es sorprendente: en las listas de la primera, algunos muchachos citan casi exclusivamente al Señor de los anillos. En la muestra del 2002 se obtiene una proporción de exactamente $3 / 4$ muchachos y de $1 / 4$ muchachas (33 muchachos y 11 muchachas responden la pregunta abierta ¿cuáles son los últimos libros leídos?). Entre las dos encuestas, han evidentemente tenido lugar las adaptaciones cinematográficas de Peter Jakson, con el éxito que se le conoce: un éxito mediático puede así perturbar los "gustos" de lo cual se ve que no es una cosa que tenga que ver con una naturaleza, femenina o masculina. Pero, las diferencias de sexo son tenaces, y no ceden fácilmente.

El caso del Señor de los anillos ofrece entonces una ocasión especialmente propicia para el estudio de las recepciones de género $S i$ su público evoluciona, eso no niega de ninguna manera, a priori, la diferenciación de las recepciones. Ver un mismo filme, leer un mismo libro no dice nada en efecto de la forma en que se lo ve, o que se lo lee. Una muchacha y un muchacho - puesto que es ésta la problemática que nos interesa -, delante del mismo filme, no van inevitablemente a retener los mismos recuerdos, ni extraer las mismas emociones, las mismas reflexiones, las mismas interpretaciones. Ahora bien, en el caso del Señor de los anillos, es sorprendente observar la forma en que las recepciones de las unas y de los otros son guiadas, en particular, por las revistas destinadas a los adolescentes, y cómo esta prensa supone - y 
suponiéndolo contribuye a elaborarlo - un horizonte de espera bien específico. No se trata aquí de llevar una explicación "de texto" del filme del Señor de los anillos como tal, de los estereotipos de género que podría transportar (una comunidad de salvadores de la humanidad formada solamente por muchachos, como lo observa uno de nuestros encuestados, Florian "en fin, la trama, es la comunidad del anillo, es de hombres, pero bueno, ellos habrían podido poner a una mujer dentro" o al contrario cuestionando los tópicos (los elfos tienen el cabello largo y llevan joyas art-déco, y el Nazgul es nivelado por belicoso, cuya feminidad, lejos de ser una debilidad, es de hecho el arma última)." Se trata aquí de observar cómo, para un mismo filme, se elaboran recepciones de género, y de matizar éstas, a partir de conversaciones llevadas con adolescentes ${ }^{12}$ sobre sus recepciones "efectivas" del filme.

\section{«Liv Tyler, una verdadera princesa $»$ o la ritualización de la recepción}

La diferencia de tratamiento del filme el Señor de los Anillos según el público de las revistas es manifiesto.Las revistas destinadas a las muchachas conceden un amplio lugar a los protagonistas multiplicando las fotografías "en civil": "Orlando Bloom siempre en el centro de la acción" título de Total Stars n 3 al interior de una fotografía donde se reconoce difícilmente al elfo Legolas. Al contrario, las revistas de muchacho escogen exclusivamente las fotografías del filme. Casus Belli $\mathrm{n}^{\circ} 23$ y Lotus Noir $\mathrm{n}^{\circ} 70$ ponen en la portada una fotografía de Frodo sin precisar el nombre del personaje ni el nombre del protagonista. En las revistas "de muchachas", una amplia parte se consagra al actor, su vida privada o su carrera: : "Viggo habría encontrado a su Arwen?" (Total Stars n 3 p. 7), Orlando está enamorado (Super n 183 p. 28), "La vida según Pippin" (Total Stars $\mathrm{n}^{\circ} 3$ p. 6)." La portada del fuera de serie One sobre El Señor de los Anillos ( $\left.\mathrm{n}^{\circ} 7\right)$ anuncia "las confianzas de los protagonistas" y la revista pone en paralelo sobre una página doble a los personajes del filme y a los actores comparando sus fotografías, su edad, su tamaño así como las razas de los personajes de la trilogía y las nacionalidades de los actores (p. 4-5).

Estas revistas cultivan dos grandes temas en torno al Señor de los Anillos: la identificación y la emoción. Los actores frecuentemente son confundidos con su papel en la trilogía. Liv Tyler (Arwen) es cualificado, en portada de Total Stars n 3, de "verdadera princesa": "joven casado romántico, diáfano y misterioso, se llega a confundirse con su personaje Arwen en la trilogía del Señor de los Anillos." :Dotada con una risa cristalina la cual utiliza a menudo, cómo no agrietarse delante de tanto de gracia y de belleza? (Total Stars n 3 p. 20). Orlando Bloom (Legolas) dice haberse "transformado en Elfo": "bebía tisana con limón." (One n’ 25 p. 28). Viggo Mortensen (Aragorn), durante el rodaje, "caminaba con los pies desnudos" y "prefería guardar para él mismo la tenida de Aragorn incluso cuando él no debía grabar" (Total Stars n 4 p. 13). Elijah Wood (Frodo) se siente "cerca de los Hobbits": "como ellos, me gusta tener a mis amigos en torno mi, aprecio la

12. No se trata más que de pistas de reflexión, elaboradas sobre una decena de entrevistas focalizadas a adolescentes entre 12 y 17 años. 
buena comida y hacer la fiesta”. Esta definición transfiere incluso a la incorporación, puesto que según Orlando Bloom, "la mayoría de los protagonistas del Señor..." se hicieron tatuar "un símbolo "elfique" que representaba el 9" (Super n' 183 p. 28)."

La insistencia sobre los sentimientos en estas revistas es notable. De manera general, las entrevistas hacen hincapié en la emoción: "El Retorno del rey es el filme más triste de la trilogía." Lloré mucho cuando lo miré" (Dominic Monaghan, Total Stars n 3 p. 18), "es un filme muy bello y emocionante" (Elijah Wood, Total Stars n 3 p. 16). Los actores agregan en la misma entrevista: "soy extremadamente romántico. Creo firmemente en el gran amor. Yo adoro mirar filmessentimentales, ¡ellos me hacen llorar como un bebé; Yo No puedo impedirlo, soy seguramente demasiado sensible, pero es como eso". Luego, tanto en las entrevistas como en las cartas de los lectores (¿lectoras?), el gran asunto es el amor: "Yo me llamo a Angéline y tengo 14 años. Soy super mega fans del super Elijah Wood. Yo lo encuentro demasiado bello" (One n' 25, p. 70), "Yo me llamo a Pauline y tengo 14 años. Te escribo porque soy fans de Orlando Bloom. Yo lo amo demasiado. ¡Este tipo es demasiado genial! Además de ser el hombre más bello de la tierra, el actúa demasiado bien, él tiene el aire súper simpático $y$ divertido" (One n 25, p. 71$)$."

Así en el tratamiento del filme, las escenas de amor entre Arwen y Aragorn son puestas al inicio. El número extraordinario de One (No 7) ofrece una ficha representando el beso entre Arwen y Aragon en el filme. El número extraordinario No 2 de Magic people sobre "La magia del Amor" pone en portada una foto de Arwen y Aragorn sub- titulada: "el amor divide". Comparativamente a su tiempo de aparición en el filme, el lugar mismo acordado a Liv Tyler (por artículos, fotos ... ) es desproporcionada "Arwen es sincera, ella no es más que lealtad y amor", señala Liv Tyler. "Ella me ha permitido jugar escenas muy fuertes en emociones como cuando ella se encuentra con Aragón, el hombre de su vida" (Total Stara No 3 p. 20). Para el último aspecto de la historia, la producción difundió siete afiches de los cuales cinco son consagrados a un único personaje: así, hay un afiche para Aragorn, Frodo, Gandalf, Gollum y Arwen. Obviamente, este personaje femenino personifica el aspecto "romántico" del filme quien debe atraer a un público femenino. Por otra parte, Liv Tyler es la única que aparece en las revistas para adolescentes en su traje de Elfa, para preservar su Áurea maravillosa en el imaginario de las jóvenes muchachas ${ }^{13}$.

En las revistas de juegos de rol o de video juego, que dan también una gran cobertura al Señor de los Anillos, Arwen pierde su papel preponderante. Casus Belli n 23 consagra un largo expediente sobre la adaptación del filme en juego de rol (p. 14-21), ilustrada de numerosas fotografías del filme (15 en totalidad) pero Arwen allí no aparece. Según el periodista, el "romance con Arwen" debe servir solamente para humanizar y dar vida al personaje de Aragorn (p.15).En este tipo de revista, las referencias se sacan solamente del universo de Tolkien, a través de los juegos, los libros y el filme. Los actores muy raramente son nombrados por su nombre

13. Por otra parte, Arwen debía participar en la batalla del pozo sin fondo de Helm (Las Dos Torres) y combatir de lado de Aragorn. La idea fue abandonada (por fidelidad al libro, según One fuera de serie n 7 p. 18): eso crea una dicotomía entre Arwen, personaje diáfano y romántico, y Eowyn, belicoso descarado y sin miedo. 
civil y allí no hay ninguna fotografía de los actores fuera del marco del filme. Por contrario, el nombre de Tolkien es omnipresente, incluso cuando es el filme lo que se menciona: " $\mathrm{El}$ Retorno del Rey, se lo conoce sin haberlo visto: el será bello, magnifico, muy adaptado con relación al libro, e iremos a verlo dos veces sin interrupción" (Backstab n 46 p. 6). Así, la lectura del Señor de los Anillos se presenta como una evidencia mientras que en las revistas para adolescentes, la lectura del libro no es un preliminar requerido. En One, Orlando Bloom afirma: "que usted haya o no leído el libro, es una historia espléndida y, cuando vaya a verla al cine, usted se apropiará de una pequeña parte" ( ${ }^{\circ} 25$ p. 28). Elijah Wood reconocen en una entrevista nunca haber terminado la trilogía: "escuché mucho a aquellos que habían leído los tres volúmenes con el fin de recoger un máximo de informaciones" (Total Stars n 3 p. 16); según Total Stars n 4, Viggo Mortensen no leyó la trilogía antes de hacer el filme (p. 12) y David Wentham (Faramir) reconoce, "[ su ] gran vergüenza”, que él había escuchado la trilogía de Tolkien y que él mismo había ensayado de leerla: "pero se había detenido en algunas páginas ya que no llegaba realmente a sumergirse en esta historia que encontraba muy complicada [... ] pero, después, llegué hasta el final “(p. 16). En estas revistas subyace que leer la trilogía no es obligatoria, basándose en un horizonte de lectura supuesta: los adolescentes son escasos lectores del Señor de los Anillos."

Se ve así que estas revistas tienden e intentan, entonces, guiar las recepciones de sus lectores asociando muchachas y a muchachos a universos diferentes. Antes de ver cómo las recepciones efectivas matizan las recepciones previstas, se puede observar que estas revistas no presentan un lector monolítico. Las jóvenes pueden leer revistas de muchachos como lo muestra la carta de "Zoé, 13 años y medio" en el correo de los lectores de Lotus Noir (n 70). Al contrario, "tres amigos, Jess, Vaues y Thomas" escriben al correo de lectoras de Total Stars $n^{\circ} 4$ para pedir un número extraordinario sobre la trilogía: "Encontramos éste filme brillante, tanto como a sus actores (sobre todo Viggo, Elijah y Orlando)". "'Podrías darnos sus direcciones?" (p. 65).

\section{De las recepciones queridas a las recepciones efectivas}

Como lo muestran estos ejemplos de "tránsfugas de género", las diferencias caricaturescas entre géneros que vehiculizan las revistas no puede traspasarse de manera automática a la recepción o al comportamiento de los individuos. Como lo recuerda Eric Maigret (1995): "la presencia de "estereotipos" no da ninguna indicación sobre la relación que se mantiene con ellos".

Todos los adolescentes interrogados, cualquiera que sea su edad, su origen social y su sexo, tienen un conocimiento claro de los estereotipos de género. ¿Las respuestas a la cuestión del Señor de los Anillos, nos dirá más bien que es para las muchachas, para los muchachos, o para los dos? son así elocuentes:

- Bueno es más para los muchachos, yo pienso... porque allí no hay realmente una historia de amor, de parte de Arwen, y Eowyn y todo... pero bien, jellos han escogido a buenos actores también para complacer a las muchachas también! (Emilien, 14 años, padre cuadro superior, madre mujer al hogar y artista pintora independiente). 
-Yo pienso que es para los unos como para las otras, ¡Ahi, porque hay allí... hay tanta acción que... y hay una historia de amor dentro pues eso, lo que le puede agradar realmente a todo el mundo. (Eric, 15 años, padre electricista y madre comerciante)

-Bueno para los dos; allí hay ciencia ficción y violencia para los muchachos y sentimiento para las muchachas (Amy, 12 años, padre artesano, madre enseñante).

- eso depende... ¿ieso depende de los gustos que... para mí, yo veo que hay filmes, que agradan a amigos, yo les dice, tu aprecias eso? porque es mi estilo... ¿̇la trama, no sé, yo diría, quizá, que es más para los muchachos..

- por qué?

- bien todo lo que es trifulca, pero bueno, hay allí todo lo que es psicología, por eso, por todo eso, aquí... (Florian, 17 años, padre jefe de empresa de restauración desempleado, antiguo manipulador, madre profesora de inglés)

Así pues, los muchachos irían a ver (o a leer) al Señor de los Anillos por las escenas de batalla mientras que las muchachas serían atraídas por las escenas de amor. Pero las entrevistas revelan una recepción más matizada, sobre todo por las adolescentes. Sara (14 años, padre analista programador y madre enfermera) reconoce sin vergüenza preferir las escenas de batalla y adorar los filmes de horror, "los filmes donde hay fantasmas, trucos y donde no hay más que vampiros, hombres- lobos...” . Amy dice, también, adorar la escena de batalla. Varía el grado de explicitación de esta atracción a- estereotípica. Mientras que Sara apenas justifica su elección ${ }^{14}$, Amy lo garantiza por un juicio estético (y moral): :"- Tu haz tenido una escena preferida?" - Era... aquella... durante la guerra, con los elefantes. ¿- Y por quése gustó? - Porque era... de los grandes elefantes, y de las pequeñas personas que llegaban a hacer caer a los grandes elefantes."

La crítica femenina de los estereotipos de género emana principalmente de las adolescentes de los medios más pudientes, para quienes funciona como un medio de distinción:

- ¿Y la historia de amor, es un aspecto del film que te gustó? -Sí, me gustó, pero a veces encontré que era un poco demasiado largo. (Carole, 12 años, padre ingeniero, madre jefa de proyectos).

¿tú te acuerdas del nombre de los personajes? - Luego, de algunos, pues todo el mundo no se detiene más que a hablar de eso .... Entonces, allí está Legolas. (Risas.) - ¿Por qué te ríes? - Bueno porque todo el mundo... en fin, las direcciones de e-mail es legolasnanana. (...) las muchachas, no dejan de decir «él es demasiado bello». Lo encuentro demasiado feo pero bien... (Amy).

Bien para los dos, aunque algunas muchachas que yo diría, con maldad mimadas no aprecian el filme. i-Tu has hablado de eso con muchachas? - Bien... de las muchachas que están en mi

14. Cuando se le pregunta ipor qué ella ama esta escena, Sarah responde simplemente : « yo no lo sé, me gustan los trucos donde hay acción $\gg$. 
clase, ellas dicen: ¡ohi, es violento, nana (Lulla,13 años, padre ayudante de laboratorio y madre investigadora en el ámbito de las vacunas).

Bien para los dos, bien

De la misma manera, ambos- donde la madre tiene más diplomas que el padre- critican al personaje de $\operatorname{Arwen}^{15}$.

Arwen, bien, ella pasa, a pesar de todo, bien, un buen filme, y a medio lloriquear, pero Eowyn, es una luchadora y todo, ella da un puntapié a las nalgas de los hombres, participa verdaderamente en la batalla de la última alianza, está bien... (Lulla)

ella, con los cabellos marrón, largos... cuando ella decía yo voy a morir todo eso, y bien entonces, muero! (Amy)

Pero la vehemencia de estas palabras no debe generar una ilusión. Así, a la pregunta “¿cuál es tu escena preferida?" Lulla emite un juicio estético: "mi escena preferida, es ... cuando, cuando se.. en el hecho, es la relación entre dos imágenes, es cuando se ve la imagen de lo que la Comarca hubiera podido llegar a ser si los anillos no hubiesen sido destruidos, todo quemado y todo, y cuando se la ve toda radiante al fin del filme, y la pequeña hija de Sam que sale..." Pero es un juicio de otro orden- $y$ contradictorio- quien aflora para la escena menos amada:

« La escena de amor entre Aragorn y Arwen, por que Aragorn, yo no puedo verlo.- ¿A sí? ¿Y por qué?- Porque yo encuentro que él es pretensioso, sucio y apestoso.- ¡Ah, bien, dicho entonces; ¿y en el libro? -Bien

Lulla, gran lectora y coleccionista de las revistas estudiadas más arriba, testimonia así una ligazón a los códigos femeninos del "bello matrimonio". Es así, menos el estereotipo mismo que la inadecuación de su representación lo que molesta a Lulla: el príncipe hubiese debido ser más encantador.... De la misma manera, Amy justifica su rechazo de Legolas por el hecho de no amar las rubias, y declara como filme preferido Retorno a Cold Mountain, reciente remake de Tanto se lleva el viento: Bien, allí había sentimiento, no mezclado con la ciencia ficción, y el fin es un fin triste, más que lo habitual, que es el final feliz, y se sabe desde el inicio lo que va a pasar, entonces desde allí, uno se pregunta sí el va a sobrevivir, etc."

Marina (17 años, primer año de secundaria, padre ingeniero y madre jefa de proyectos) tiene igualmente una actitud ambigua frente al estereotipo. De un lado, ella rechaza la historia de amor ("Quizás ellos se sentían un poco obligados de poner una historia de amor, pues en todos los filmes hay eso..- ¿Y porque ellos lo agragarían?- Bien, por los jóvenes..los jóvenes.. ¿Y 
tú, eso te hubiera faltado, te hubiese faltado que no hubiera una historia de amor?- Bien, yo lo hubiera notado, porque ... no es lo habitual, ipero sin historia de amor, eso hubiera sido bien, inclusoj) pero ella prefiere a la más femenina de los personajes ...” yo he bien amado a EO ... - ¿La rubia, Eowen?- No, la otra, la morena.- ¿Arwen, la elfo?- Sí, sí, yo encuentro que ella actuaba mejor.. y yo no sé, la rubia, yo siento que.. ella es menos amada, enfin, sí, esta era una heroína, pero al mismo tiempo ella no lo es tanto, ella no tiene la misma imagen que Arwen”. ¿Así, el personaje de Eowyn sufriría de ser menos femenino?

La aceptación de los estereotipos parece menos problemática en los muchachos. Todos los adolescentes interrogados dicen apreciar el filme por las escenas de batalla y por la historia en general, refiriéndose así explícitamente al modelo clásico de las preferencias masculinas:

¿Por qué te gusta el filme ?- Bien, hay combates, yo amo los filmes de acción.. y ¡He aquíi (Yann, 15 años, padre librero y madre cajera)

Es un poco tonto, los combates a la espada, y todo, yo amo bien (eso).... (Emilien)

- Bien yo gusto de las grandes batallas. Me gusta mucho eso. Me gusta ver las masas de... bien esto es un poco, tal vez, típico para... más para el hombre que para la mujer... No sé.. - ¿por qué dices eso? - bien, no sé, quizá la fascinación de las armas, es más marcante para el hombre que para la mujer... (Philippe, 17 años, padre cuadro de una gran empresa, madre profesora de alemán).

- Bien y luego las escenas de bastón... (Florian)

Mientras que es "vergonzoso" para una adolescente afirmar su adhesión a los estereotipos femeninos (como el de la bonita princesa y su príncipe encantador), parecería casi "vergonzoso" para un adolescente no afirmar su adhesión a los estereotipos masculinos, por no decir a los estereotipos de la virilidad. Aquí aún, bajo una forma disfrazada, se manifestaría una determinada forma de soberanía masculina: el modelo reconocido es el de la virilidad, versus la zalamería femenina. Precisemos obviamente que este estereotipo se revela tan vinculante para los muchachos como para las muchachas.

En efecto, las recepciones no son monolíticas. Así pues, Florian y Philippe dan prueba ambos de su emoción, más o menos fácilmente aceptada y "reconocida”:

$i$ - qué es lo que tiene una escena que te ha marcado?

- sí, (inmediatamente, sin reflexionar) en la 1 , cuando se hacen atacar, me acuerdo más del nombre de los personajes, exactamente en el 1, y que Frodo decide ir completamente solo, exactamente en 1, francamente esta escena allí, ella es... muy estimulante, emocionante. - ¿y qué es lo que te conmovió? - bien, yo no sé, de ver cómo los hombres llegan a darse cuenta de sus errores, y deciden cambiar... yo encontré eso muy bien - ¿Entonces, más a nivel de la psicología?sí, sí, he aquí. ¿¿Bien y luego las escenas de pelotera... (...) - sí,ż no es la pelotera por la pelotera? - ah sí, queda claro, si no, no es interesante. (Florian)

-finalmente, sobre todo hacia el final, con los barcos, todo eso, sobre todo eso que está lleno de emoción, se está un poco... se está como eso (enrojece bajando la cabeza). - ¿Entonces, la 
emoción, tú la haz experimentado? ¿- Bien sí, sí, sí... (...)- ¿Y cuándo dices la emoción, tu tenías la garganta apretada, de lágrimas? ¡por último, yo te digo eso, yo lloré, ¡eih! i- No, no, no hay lágrimas, pero... sí a pesar de todo, la garganta apretada, porque... bien, y luego se no sé, se espera, pero Frodo parte con! - ¿y eso lo aprecias? - Sí, sí, me gusta... z- Y con tu amigo, esto era similar, cuando hablaban? - Bien, yo no sé si eso era como eso con él, no se ha hablado de éso... $i^{-}$Y ustedes hablaban más bien de las trifulcas? -Sí, de las trifulcas, no... no de eso (aire un poco molesto). - Tiene, a pesar de todo, reticencia a hablar... - Sí... con amigos como eso, no. Por último, eso depende de los amigos... a mí eso me es igual, pero es por sobre todo de su manera sí eso los interesa o no, todo depende cómo ellos reaccionarían al respecto... (Philippe)

La atención acordada a la psicología emana principalmente de las adolescentes de medios acomodados ${ }^{16}$.

El lugar concedido a Gollum, el personaje ambiguo de la trilogía, hombre decaído físicamente y dotado de una doble personalidad, es aquí luminoso: personaje preferido para la mayoría de los adolescentes de origen acomodado (que lo encuentran "divertido"), y al contrario rechazado por los adolescentes de origen desfavorecido... Pero la cuestión queda por resolver, si la diferenciación social actúa en la recepción de las emociones, o en su grado de manifestarlo con palabras, en el sí, en el amigo... y en la encuesta ${ }^{17}$.

Se ha visto de éstas entrevistas, que los muchachos y las muchachas reaccionan de diferente manera frente a los estereotipos: si las muchachas, sobre todo cuando proceden de medios acomodados, se distinguen por un rechazo violento de lo "superficial" (lo que no les impide pronunciar juicios estéticos sobre tal o cual protagonista), los muchachos, por su parte, asumen sonriendo el gusto "tonto" por las "trifulcas" (lo que no les impide tener la garganta apretada en algunas escenas)... La consecuencia de este trabajo, al seguir llevando entrevistas con adolescentes, tiene por objeto delimitar de más cerca el concepto de "psicologización" que, lo vimos, no se expresa de igual manera según el medio social: ise trata de recepción, o de expresión? Si la variabilidad de las recepciones de una misma obra según el género, la nacionalidad, etc., de los individuos ha inspirado numerosos trabajos de sociología de la recepción -p.e. Burgos et al, 1982; Flynn, 1986- y también estrategias de marketing ${ }^{18}$, es necesario restituir los matices, los juegos, los acuerdos con las normas que elabora cada uno, lejos de la ley monolítica del género, que resumiría, transponiéndolo, el epígrafe del Señor de los anillos: "un anillo para a todos controlarlos, [... ] y en la oscuridad a todos vincularlos".

1. Eric Maigret habla así de la «psicologisación » creciente de las clases medias y superiores.

2. Las entrevistas van a ser mezcladas con adolescentes desfavorizados para completar este punto.

3. Martin Baker conduce actualmente un trabajo referido al Análisis de las estrategias de promoción del Señor de los Anillos, contruido a partir de « paratextos » : según los países y sus « características » supuestas, los afiches, las bandas de anuncio, etc.., las cuales no son las mismas. 


\section{Referencias bibliográficas}

Détrez, C. (jun. 2003). "Vues à la TV: Nana, Cosette, Esmeralda et les autres”. Réseaux N¹77.

Donnat, O. (1997). Pratiques culturelles des Français. Enquête 1997. Dep. La Documentation Française. Baudelot, C. ; Cartier, M. ; Détrez, C. (1999). Et pourtant ils lisent. Paris: Seuil.

Maigret, E. (abr. 1995). « 'Strange grandit avec moi'. Sentimentalité et masculinité chez les lecteurs de bandes dessinées de super-héros». Réseaux $\mathrm{N}^{\circ} 70$.

Burgos, M.; Josha, P. ; Leenhardt, J. (1982). Lire la lecture. Paris: Editions Le Sycomore.

Flynn, Sch., ed. (1986). Gender and reading. London: The Johns Hopkins University Press. 